
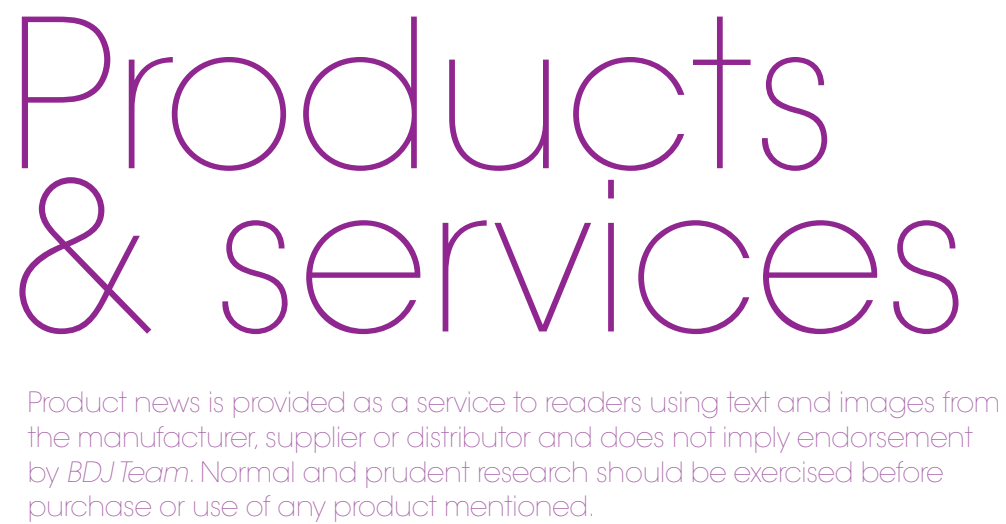

\title{
YOUR PARTIAL DENTURE PATIENTS ARE ALMOST TWICE AS LIKELY TO LOSE MORE TEETH
}

Wearing a partial denture almost doubles the patient's risk of losing more teeth than non-partial wearers.

Tooth loss can have a profound effect on the lives of patients both physically and emotionally $-45 \%$ of patients experience difficulties accepting the loss of their teeth. ${ }^{2}$ As their trusted dental professional, your advice can have a huge impact on a patient's wellbeing, whilst also helping them to avoid losing more teeth.

What's more, as the population ages, and the chance of wearing a denture increases with age, ${ }^{3}$ you will be seeing more denture patients on a day-to-day basis. Within the next 35 years, it is expected there will be 2 billion people

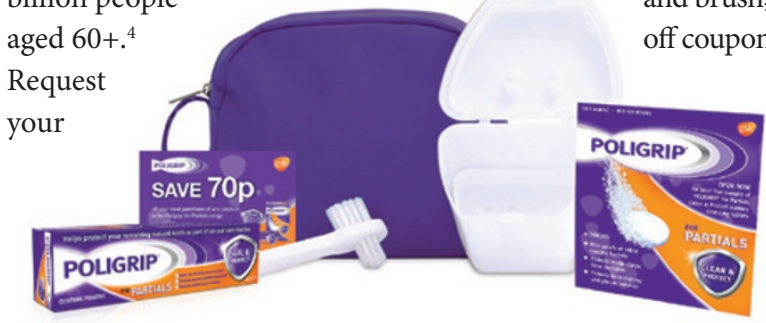
2011: 29: 71 1-719.

2. Davis DM et al. Br Dent $J$ 2000; 188: 503-506

3. Steele JG et al. Br Dent J 2000; 189: 598-603

4. UNFPA \& HelpAge International, 2012

\section{MAKE A GREAT FIRST IMPRESSION}

Delivering solutions for the double-mix, putty-wash and monophase techniques. Aquasil Ultra is a quadrafunctional, hydrophilic A-silicone impression material with excellent wettability, high tear strength (at least 40\% stronger when compared with 23 other leading wash materials, according to in-vitro tests) and dimensional stability. Designed to minimise distortion, Aquasil Ultra reliably captures and retains the finest detail, minimising remakes and saving valuable time and money for clinicians, technicians and patients alike.

To find out more about Aquasil Ultra contact DENTSPLY UK on 08000723313 or visit www. dentsply.co.uk

To learn about effective techniques

for impression taking visit www.

dentsplyacademy.co.uk and access the FREE CPD webinar

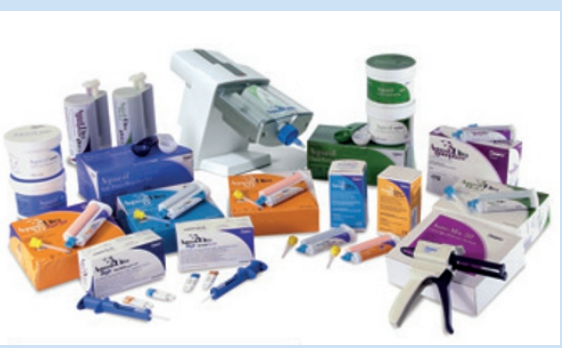

CELEBRATING INITIAL MILESTONES

\section{After only a couple of months since the announcement of its launch The European Aligner Society (EAS) is already celebrating several exciting milestones as it progresses towards fulfilling its initial aims. The Society was created to provide continuing information about developments in clear aligner therapy, and develop a strong backup to the formal training that takes place at postgraduate level}

As an over-arching members' Association. EAS aims to provide membership services which will form the basis of the 'aligner' community: provide support to members: offer advice and guidance; and make resources available to support and develop education in clear aligner therapy. This is an invitation to join us and be involved with an Association which is solely for aligner providers and those interested in becoming aligner providers

\section{Landmarks}

The EAS will formally launch at the World Federation of Orthodontists Congress at Excel in London between 27-30 September, 2015. Potential members are invited to visit stand 548 to meet the Society founders and sign up.

It has just been announced that the EAS inaugural conference - Aligners, a clear new era in orthodontics - will take place in Vienna on 13-14 February, 2016. The directors of the Society have put together a programme of lectures and workshops led by some of the world's foremost aligner exponents. A full programme will be available at the WFO meeting.

Membership of EAS is open now. As an incentive to clinicians to support EAS as early as possible, subscription for the first year will only require renewal a year after the formal launch - September 2016. Visit www.eas-aligners.com and join the Society on an ever expanding aligner journey.

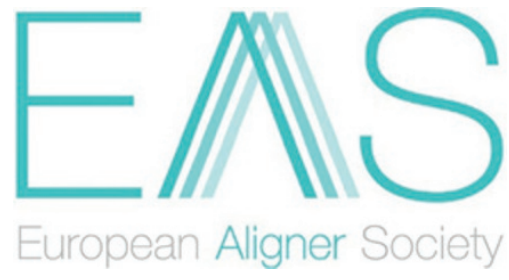

If you would like to promote your products or services direct to the dental industry in BDJ Team, call Andy May on 02078434785 premaila.may@nature.com. 